nephron

Practice
Nephron 2015;131:221-226

DOI: $10.1159 / 000441425$
Received: April 16, 2015

Accepted after revision: September 30, 2015

Published online: November 11, 2015

\title{
Guidelines for Classification of Acute Kidney Diseases and Disorders
}

\author{
Rebecca Barry Matthew T. James \\ Department of Medicine and Community Health Sciences, Cumming School for Medicine University of Calgary, \\ Calgary, Alta., Canada
}

\section{Key Words}

Acute kidney injury · Chronic kidney disease · Classification clinical characteristics, incidence, and prognosis of AKD, as well as the implications of case identification based on the AKD criteria.

(c) 2015 S. Karger AG, Basel

\section{Definition and Classification of Acute and Chronic Kidney Diseases}

Over the last two decades, substantial efforts have been made to standardize definitions and classification systems for kidney disease. In 2002, a guideline for chronic kidney disease (CKD) was published by the Kidney Disease Outcome Quality Initiative (KDOQI), which established a definition for CKD based on reduced estimated glomerular filtration rate (eGFR) and albuminuria for at least 3 months, and a 5-stage classification system based predominantly on eGFR [1]. Subsequently, modi-

Contribution from the AKI and CRRT 2015 Symposium at the 20th International Conference on Advances in Critical Care Nephrology, Manchester Grand Hyatt, San Diego, Calif., USA, February 17-20, 2015.

\section{KARGER 125}

(c) 2015 S. Karger AG, Basel

$1660-8151 / 15 / 1314-0221 \$ 39.50 / 0$

E-Mail karger@karger.com

www.karger.com/nef
Dr. Matthew T. James

Foothills Medical Centre

1403 29th St. NW, Calgary, AB T2N 2T9 (Canada)

E-Mailmjames@ucalgary.ca 
fications to the CKD staging system were published in the Kidney Disease Improving Global Outcomes (KDIGO) Guidelines for Evaluation and Management of $\mathrm{CKD}$, which included further staging based on the presence of albuminuria and recognition of the cause of kidney disease [2].

There has been a similar evolution in the definition and classification of acute kidney injury (AKI). In 2004, the Acute Dialysis Quality Initiative first proposed the Risk, Injury, Failure, Loss and End-Stage Renal Disease (RIFLE) criteria for diagnosis and classification of acute impairments in kidney function, which included five stages ranging from small changes in kidney function or urine output, to kidney failure and end-stage renal disease [3]. These criteria were subsequently refined into a 3 -stage system, and further disseminated by the Acute Kidney Disease Network in 2007 [4]. In 2012, the KDIGO Clinical Practice Guideline for AKI consolidated these criteria into the most recent definition and classification system for AKI [5]. The current definition and classification of AKI relies upon functional criteria including changes in serum creatinine (SCr) and urine output, whereas the definition for CKD incorporates both functional criteria (i.e. eGFR) as well as structural markers of kidney damage (e.g. albuminuria) [2, 5].

\section{Benefits and Limitations of Current Definitions and Classifications}

The current definitions of AKI and CKD are generally well founded, based upon strong, graded relationships between acute changes or sustained reductions in laboratory measures of kidney function with important clinical outcomes of AKI and CKD, respectively [6]. Established definitions and classification systems for AKI and CKD have led to conceptual models that are increasingly applied in clinical practice, research, and public health [2]. Benefits have included enhanced communication, recognition, and awareness of kidney disease among clinicians [7]. The dissemination of established definitions for both AKI and CKD has also helped to foster further clinical research on both disorders $[2,8]$.

Nonetheless, the current definitions and classification systems have limitations. The CKD definition based on reduced eGFR resulted in high estimates of prevalence of the disorder, particularly in the elderly, raising concerns about over-diagnosis, unnecessary referral and treatment, and economic burden $[9,10]$. For AKI, there may be substantial heterogeneity in case identification and prognostic relevance of the AKI staging system depending on whether it is based on SCr or urine output criteria [11]. The identification of AKI using current SCr-based definitions may be delayed because changes in creatinine are a late marker of kidney damage [12]. There have also been concerns about the clinical relevance of applying small changes in $\mathrm{SCr}$ to define AKI among patients with advanced CKD [13].

Further limitations to the current AKI and CKD definitions and classification systems arise from the fact that there are patients who present with alterations of kidney function and structure that do not meet the established criteria for AKI or CKD. This is not surprising because the definitions and classification systems were developed by different groups focusing on different clinical syndromes. Nonetheless, abnormalities of kidney disease markers that do not meet existing definitions for AKI and CKD still require medical attention to avoid adverse outcomes. For these reasons, the KDIGO 2012 Clinical Practice Guideline for AKI Guidelines work group proposed an operational definition for acute kidney diseases and disorders (AKD) along with an integrated approach for its identification alongside AKI and CKD [2]. The work group proposed that this new definition and classification system could be applied to clinical approaches to patients with abnormalities of kidney function and structure that encompassed both AKI and CKD, as well as those individuals with abnormalities not captured within current definitions.

\section{Proposed Criteria for AKD}

The proposed criteria for AKD encompass the definition for AKI, but may also be defined by a glomerular filtration rate $(\mathrm{GFR})<60 \mathrm{ml} / \mathrm{min} / 1.73 \mathrm{~m}^{2}$ for $<3$ months, a decrease in GFR by $\geq 35 \%$ for $<3$ months, or an increase in SCr by $>50 \%$ for $<3$ months (table 1 ). The approach that may be taken to identify patients with AKD is illustrated in figure 1. Patients with no prior kidney disease or with preexisting CKD can develop AKD. A patient is considered to have AKD when, compared to SCr measurements at least 3 months prior, they present with a new or previously unrecognized decrease in GFR or increase in SCr. Should repeated SCr measurements show that SCr is increasing (or GFR decreasing), patients with $\mathrm{AKD}$ may be further classified as having AKI if the change in SCr is $>26 \mu \mathrm{mol} / \mathrm{l}$ or a 1.5 -fold increase within a 7 -day time frame, consistent with the current KDIGO definition of AKI. Patients may meet the definition for AKD without 


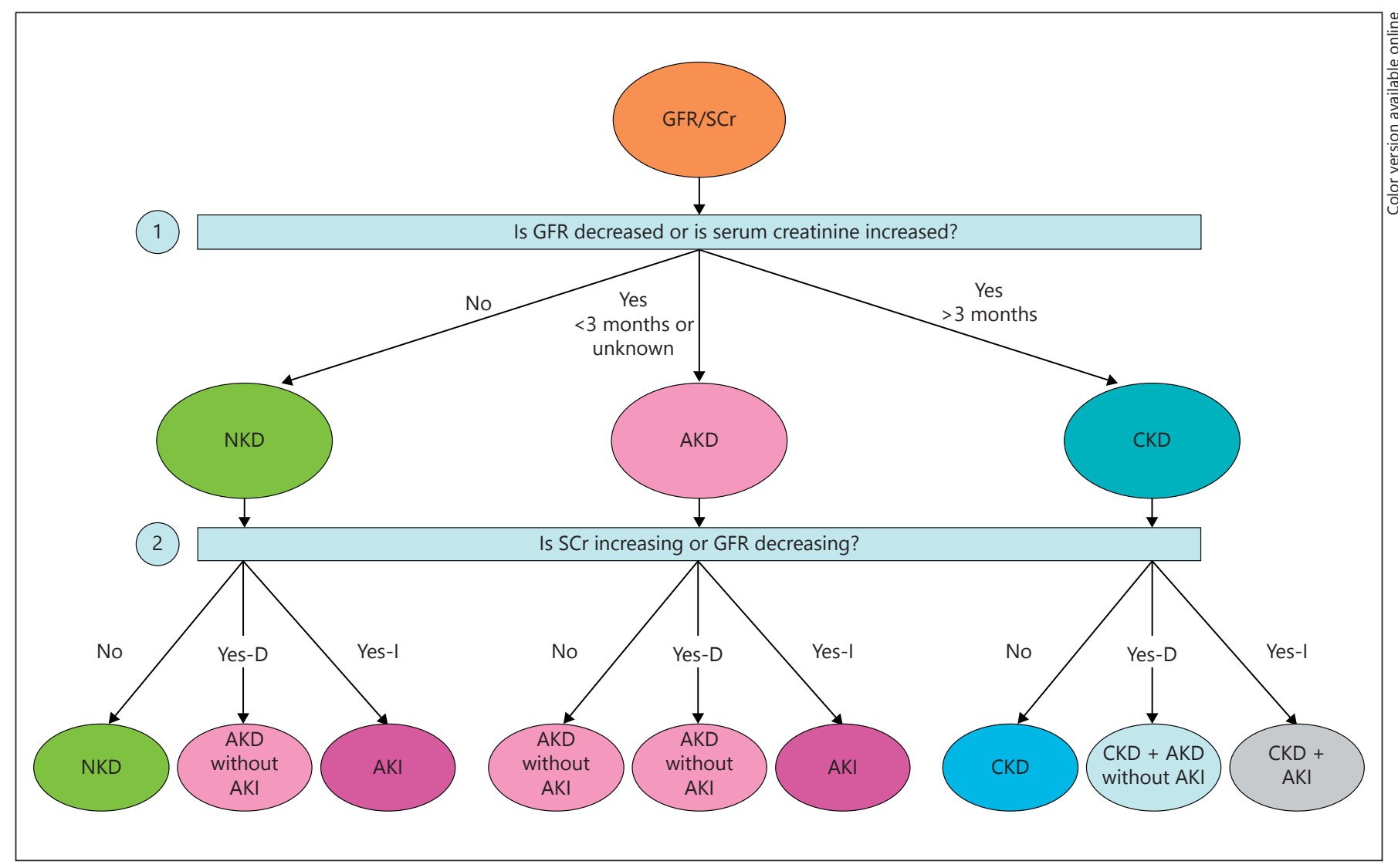

Fig. 1. Diagnostic approach to alterations in kidney function and structure. Adapted from [5]. NKD = No kidney disease; Yes-D = change in SCr meet AKD criteria but not AKI criteria; Yes-I = change in SCr meets AKI criteria.

Table 1. KDIGO definitions of AKI, CKD and AKD

\begin{tabular}{lll}
\hline & Functional criteria & Structural criteria \\
\hline AKI & $\begin{array}{l}\text { Increase in SCr by } 50 \% \text { within } 7 \text { days, or increase in } \\
\text { SCr by } 0.3 \mathrm{mg} / \mathrm{dl}(26.5 \mu \mathrm{mol} / \mathrm{l}) \text { within } 2 \text { days, or oliguria }\end{array}$ & None \\
\hline CKD & GFR $<60 \mathrm{ml} / \mathrm{min} / 1.73 \mathrm{~m}^{2}$ for $>3$ months & Kidney damage for $>3$ months \\
\hline AKD & $\begin{array}{l}\text { AKI, or GFR }<60 \mathrm{ml} / \mathrm{min} / 1.73 \mathrm{~m}^{2} \text { for }<3 \text { months, or decrease in } \\
\text { GFR by } \geq 35 \% \text { or increase in } \mathrm{SCr} \text { by }>50 \% \text { for }<3 \mathrm{months}\end{array}$ & Kidney damage for $<3$ months \\
\hline NKD & GFR $\geq 60 \mathrm{ml} / \mathrm{min} / 1.73 \mathrm{~m}^{2}$ for $<3$ months stable $\mathrm{SCr}$ & No damage \\
\hline \multicolumn{2}{c}{ Adapted from $[5] . \mathrm{NKD}=$ No kidney disease. } & \\
\hline
\end{tabular}

meeting the criteria for AKI, thereby being classified into a group with kidney disease previously unacknowledged by the preexisting AKI and CKD definitions. Patients with preexisting CKD may also meet the criteria for $\mathrm{AKD}$, with or without AKI (fig. 2).

Guidelines for Classification of AKD

\section{Initial Responses to the AKD Concept}

Since the KDIGO 2012 Clinical Practice Guidelines for AKI first proposed the criteria for AKD, there have been two responses to these guidelines that specifically com- 


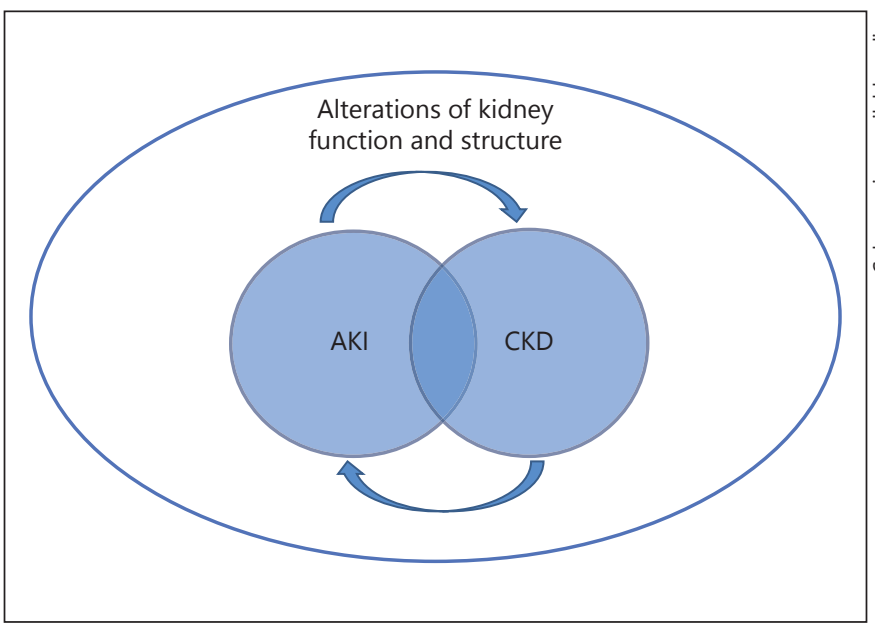

Fig. 2. Relationships between $\mathrm{AKI}, \mathrm{CKD}$, and $\mathrm{AKD}$. AKD encompasses a spectrum that includes both AKI and CKD. AKI may contribute to the development or progression of CKD, while CKD is a strong risk factor for AKI.

mented on the AKD concept, one from the National Kidney Foundation KDOQI [7], and another from the Canadian Society of Nephrology (CSN) [8]. The National Kidney Foundation KDOQI [7] expressed concern that the introduction of the AKD terminology may cause confusion among physicians and other clinicians and might inadvertently cause them to overlook other important diagnostic considerations when evaluating patients first presenting with kidney disease [7]. They also expressed concern that because a broad range of unique conditions may be classified under the umbrella term of AKD, clinicians may label patients with $\mathrm{AKD}$ while failing to distinguish between the underlying causes. The KDOQI commentary suggested that a more in-depth evaluation was warranted about which specific kidney diseases are likely to be captured under the AKD criteria. While they suggest that the term may be useful in epidemiologic research, this work group cautioned that it should not yet be used in clinical practice.

The commentary provided by the CSN also highlighted that although a classification scheme relevant to all patients with kidney disease was important and relevant to further research, the clinical use of the AKD criteria remained uncertain [8]. Similar to the KDOQI work group, the CSN commentary highlighted that patients with several underlying clinical syndromes including rapidly progressive glomerulonephritis, acute interstitial nephritis, atheroembolic disease, and urinary tract obstruction may meet AKD criteria. The opinions of these work groups support further epidemiologic research based on the AKD concept but cautioned against the clinical adoption of the concept at this time to avoid distraction from appropriate clinical investigations to identify the underlying causes of disease and prompt treatment.

\section{Studies That Have Applied the AKD Criteria}

A recent systematic review on detection and staging of acute CKD highlighted the lack of information on the burden of AKD [14]. To our knowledge, only one published study has to date applied the AKD criteria. Chu et al. [15] examined a case series from China that underwent kidney biopsy and had evidence of active forms of histopathological lesions. Patients with predominantly fibrotic or sclerotic lesions on biopsy were excluded. Chu et al. [15] applied available SCr measurements to classify patients based on criteria for AKI, AKD without AKI, and those with neither AKI nor AKD. Patients meeting the criteria for AKD had higher SCr levels at the time of kidney biopsy and higher peak values during hospitalization than those without AKD or AKI. Patients with AKD who also met AKI criteria had the highest peak SCr values, were the most likely to require renal replacement therapy, and had the longest hospital stays and costs. Those with AKI had the lowest levels of kidney function at hospital discharge, followed by those with AKD without AKI.

Evidence from the kidney biopsies showed differences in the distribution of histopathological lesions between those with AKI, AKD without AKI, and neither AKD nor AKI. Acute tubular necrosis was almost twice as common among patients with AKI as among those with AKD without AKI (30.8 vs. 16.0\%). Acute tubulointerstitial nephritis was more common on biopsies from patients with AKD without AKI than among those with AKI (52.0 vs. $30.8 \%$ ). The prevalence of crescentic glomerulonephritis was similar among both the AKI and AKD without AKI groups (28.8 vs. $29.3 \%)$ and thrombotic microangiopathy and combined lesions were rare in both groups. These findings suggest that patients that meet criteria for AKD may have different distributions of underlying kidney diseases than those with AKI. However, these findings should be interpreted cautiously and may not be generalizable to the full spectrum of $\mathrm{AKD}$, given the bias that may be introduced by studying only patients who were selected to undergo kidney biopsy. 
Table 2. Knowledge gaps and future research directions for AKD

\begin{tabular}{ll}
\hline Knowledge gaps & Future research directions \\
\hline AKD recognition & $\begin{array}{l}\text { Characterization of incidence of AKD across various settings (hospital, community, emergency } \\
\text { department), including those not fulfilling AKI or CKD criteria }\end{array}$ \\
\hline AKD management & $\begin{array}{l}\text { Examination of processes of care for those presenting with AKD criteria, including how patients } \\
\text { with AKD without AKI or CKD are investigated and managed in clinical practice }\end{array}$ \\
\hline Prognosis of AKD & $\begin{array}{l}\text { Population-based studies to examine incidence and progression to key outcomes such as CKD, } \\
\text { end stage renal disease, and mortality }\end{array}$ \\
\hline $\begin{array}{l}\text { AKD in relation to CKD } \\
\text { and AKI }\end{array}$ & $\begin{array}{l}\text { Research to better characterize features of AKD associated with progression to CKD rather than } \\
\text { recovery or subsequent normalization of kidney function, including better understanding of } \\
\text { influence of variability of kidney disease measurements }\end{array}$ \\
\hline $\begin{array}{l}\text { Clinical impact of case } \\
\text { finding }\end{array}$ & $\begin{array}{l}\text { Evaluation of clinical implications and costs of strategies for case identifying and clinical } \\
\text { surveillance of those with AKD, including evaluation of clinical impact of introducing the AKD } \\
\text { definition into case finding and referral practices }\end{array}$ \\
\hline $\begin{array}{l}\text { Application of classification } \\
\text { systems and models of care } \\
\text { encompassing CKD and AKD }\end{array}$ & $\begin{array}{l}\text { Determine whether education, dissemination, and implementation strategies for applying the } \\
\text { AKD criteria, in conjunction with models of care that encompass both chronic and acute } \\
\text { disorders improve short- and long-term clinical outcomes }\end{array}$ \\
\hline
\end{tabular}

\section{Future Direction}

The definitions and classifications of acute and CKDs continue to evolve. New criteria proposed for AKD attempt to provide a comprehensive nomenclature for kidney diseases, encompassing those disorders that do not meet the criteria for AKI or CKD but may still require medical evaluation and treatment. While it may be premature to adopt these criteria into clinical practice, preliminary evidence supports the hypothesis that AKD criteria identify patients with varied histopathological kidney diseases of clinical importance.

Current knowledge gaps and future research needed to inform the adoption of AKD criteria in clinical practice are provided in table 2 . The AKD criteria could be useful for research on recognition, management, and outcomes of patients who present with new evidence of kidney disease across a variety of clinical settings, including the community, emergency departments, and hospitals when additional information from serial measurements of kidney function are lacking. Conceptual approaches based on AKD criteria might help consolidate research efforts to characterize the bidirectional relationships between CKD and AKI. Future research is needed to better understand the clinical characteristics, population-based incidence, and prognosis of AKD. The potential clinical impact of case finding based on AKD criteria also needs to be explored, along with more research to ultimately determine whether the application of classification systems and models of care encompassing both acute and chronic kidney disorders lead to better clinical outcomes.

\section{Disclosure Statement}

The authors report no conflicts.

References

1 Levey S, Coresh J: KDOQI guideline: clinical practice guidelines for chronic kidney disease, evaluation, classification and stratification. Am J Kidney Dis 2002;39:43-100.

2 Kidney Disease: Improving Global Outcomes CKD Work Group: KDIGO 2012 clinical practice guideline for the evaluation and management of chronic kidney disease. Kidney Int Suppl 2012;3:1-150.

-3 Bellomo R, Ronco C, Kellum JA, Mehta RL, Palevsky P: Acute renal failure - definition, outcome measures, animal models, fluid therapy and information technology needs: the Second International Consensus Conference of the Acute Dialysis Quality Initiative (ADQI) Group. Crit care 2004;8:R204-R212.

4 Mehta RL, Kellum JA, Shah SV, Molitoris BA, Ronco C, Warnock DG, Levin A; Acute Kidney Injury Network: Acute Kidney Injury Network: report of an initiative to improve outcomes in acute kidney injury. Crit Care 2007;11:R31. 
5 Kidney Disease: Improving Global Outcomes AKI Work Group: KDIGO clinical practice guideline for acute kidney injury. Kidney Int Suppl 2012;2:1-138.

6 Levey AS, Levin A, Kellum JA: Definition and classification of kidney diseases. Am J Kidney Dis 2013;61:686-688.

7 Palevsky PM, Liu KD, Brophy PD, Chawla LS, Parikh CR, Thakar CV, Tolwani AJ, Waikar SS, Weisbord SD: KDOQI US commentary on the 2012 KDIGO clinical practice guideline for acute kidney injury. Am J Kidney Dis 2013;61:649-672.

8 James M, Bouchard J, Ho J, Klarenbach S, LaFrance JP, Rigatto C, Wald R, Zappitelli M, Pannu N: Canadian society of nephrology commentary on the 2012 KDIGO clinical practice guideline for acute kidney injury. Am J Kidney Dis 2013;61:673685.

9 Moynihan R, Doust J, Henry D: Preventing overdiagnosis: how to stop harming the healthy. BMJ 2012;344:e3502.

10 Levey AS, de Jong PE, Coresh J, El Nahas M, Astor BC, Matsushita K, Gansevoort RT, Kasiske BL, Eckardt KU: The definition, classification, and prognosis of chronic kidney disease: a KDIGO controversies conference report. Kidney Int 2011;80:1728.

1 Ricci Z, Cruz D, Ronco C: The RIFLE criteria and mortality in acute kidney injury: a systematic review. Kidney Int 2008;73:538546.
2 Herget-Rosenthal S, Marggraf G, Hüsing J, Göring F, Pietruck F, Janssen O, Philipp T, Kribben A: Early detection of acute renal failure by serum cystatin C. Kidney Int 2004;66: 1115-1122.

13 Waikar SS, Bonventre JV: Creatinine kinetics and the definition of acute kidney injury. J Am Soc Nephrol 2009;20:672-679.

14 Levey AS, Becker C, Inker LA: Glomerular filtration rate and albuminuria for detection and staging of acute and chronic kidney disease in adults: a systematic review. JAMA 2015;313:837-846.

15 Chu R, Li C, Wang S, Zou W, Liu G, Yang L: Assessment of KDIGO definitions in patients with histopathologic evidence of acute renal disease. Clin J Am Soc Nephrol 2014;9:1175-1182. 